\title{
Biomimetic Slippery PDMS Film with Papillae-Like Microstructures for Antifogging and Self-Cleaning
}

\author{
Xiaoming Feng ${ }^{1,2}\left(\mathbb{D}\right.$, Huiying Guan ${ }^{3}$, Ze Wang ${ }^{2}$, Shichao Niu ${ }^{2, *}$ and Zhiwu Han ${ }^{2, *}$ \\ 1 School of Mechanical Engineering, Jiangsu University of Science and Technology, Zhenjiang 212003, China; \\ xmfeng@just.edu.cn \\ 2 Key Laboratory of Bionic Engineering, Ministry of Education, Jilin University, Changchun 130022, China; \\ wangze18@mails.jlu.edu.cn \\ 3 College of Mechanical and Electrical Engineering, Jilin Institute of Chemical Technology, Jilin 132022, China; \\ guanhy_2005@sina.com \\ * Correspondence: niushichao@jlu.edu.cn (S.N.); zwhan@jlu.edu.cn (Z.H.)
}

Citation: Feng, X.; Guan, H.; Wang,

Z.; Niu, S.; Han, Z. Biomimetic

Slippery PDMS Film with

Papillae-Like Microstructures for

Antifogging and Self-Cleaning.

Coatings 2021, 11, 238. https://

doi.org/10.3390/coatings11020238

Academic Editor: Gianni Barucca

Received: 10 January 2021

Accepted: 27 January 2021

Published: 17 February 2021

Publisher's Note: MDPI stays neutral with regard to jurisdictional claims in published maps and institutional affiliations.

Copyright: () 2021 by the authors. Licensee MDPI, Basel, Switzerland. This article is an open access article distributed under the terms and conditions of the Creative Commons Attribution (CC BY) license (https:// creativecommons.org/licenses/by/ $4.0 /)$

\begin{abstract}
Transparent materials with antifogging and self-cleaning ability are of extreme significance for utilization in outdoor solar cell devices to alleviate the performance loss and maintenance costs. Herein, with inspiration from the anti-wetting surfaces in nature, regular papillae-like microstructure arrays (PMAs) inspired by lotus leaves were designed via a common UV lithography combined with a soft replication. Subsequently, the biomimetic slippery polydimethylsiloxane (PDMS) film (BSPF) inspired by the pitcher plant was fabricated successfully by infusing with hydrophobic liquid lubricant. The resultant surface has hydrophobic surface chemistry, a slippery interface, PMAs structure. The wettability, optical characteristic, antifogging property and self-cleaning ability of the PMAs-based BSPF were characterized experimentally. The film displays excellent optical transmittance, antireflection, antifogging, and self-cleaning properties, which is superior to the flat PDMS film (FPF). Remarkably, an average reflection of $\sim 11.3 \%$ in the FPF was reduced to $\sim 8.9 \%$ of the BSPF. In addition, after gradient spray test for $120 \mathrm{~s}$, the antifogging efficiency was close to $100 \%$ for the BSPF surface in comparison with the flat PDMS film (FPF), biomimetic PDMS film (BPF) and flat slippery PDMS film (FSPF) $(35 \%, 70 \%$ and $85 \%)$. Furthermore, we also discovered that the BSPF surface exhibited a better self-cleaning performance toward a variety of liquids than solid dust.
\end{abstract}

Keywords: biomimetic PDMS film; antifogging; self-cleaning; papillae-like microstructures; slippery surfaces

\section{Introduction}

Fogging brings problems in many different fields where materials with outstanding optical performance are required [1-7]. For example, the existence of condensation reduces the precision of microscopes and other analytical instruments. It also increases the occurrence of traffic accidents as a result of blurred sight. In addition, the absorptivity of a photovoltaic solar cell is greatly impacted by surface dirt and scattering of light. Polydimethylsiloxane (PDMS) was extensively utilized in optical areas as it has high degree of transparency and low refractivity, and excellent adhesion, these remarkable advantages enable it to be reversibly attached and detached from various types of solar cells substrate [8,9].

Natural biological surfaces have optimized their physico-chemical properties and the micro-nanometer structure for diverse features, ranging from wettability to optical performance [10-13]. Especially, natural hierarchical structures have been considered as a way to achieve superior antifogging performance, such as insects' compound eyes, butterfly wings and water strider legs [14-19]. A widespread insight for self-cleaning surfaces is the lotus leaf, which offers high water repellency [20-22]. However, the surfaces of lotus leaves can't solve such fogging problems. This is because microscale fog drops can be easily trapped in the interspaces among micro-papillae and become highly pinned [23-27]. 
Additionally, the rough surface textures on superhydrophobic surface usually decrease the transparency of optical instruments. As an alternative strategy to resolve above issues, new types of liquid-repellent surfaces inspired by the pitcher plant (Nepenthes sp.) have been developed. The liquid-wetted surface retains a mobile three-phase contact line (TCL) by fully infusing a hydrophobic lubricant on rough surface, which has been demonstrated as a high-efficiency heat-transfer substrate [28,29].

Here, inspired by lotus leaves employ PDMS as substrate, the surface topographies taking the form of a regular papillae-like microstructure arrays was designed, and combines the pitcher plant-inspired lubricant-infused "slippery" surfaces, using hydrophobic liquid lubricant as modifier. In this preparation, the SU-8 negative template was fabricated by a common UV lithography. Then the papillae-like microstructure arrays were manufactured by means of a soft replication. Finally, the biomimetic slippery PDMS film (BSPF) was fabricated successfully by infusing with Krytox 1506 lubricant. The resultant surface has hydrophobic surface chemistry, a slippery interface, PMAs structure. Subsequently, the wettability, optical characteristic, antifogging property and self-cleaning ability of the PMAs-based BSPF were characterized in detail. The prepared BSPF surface improves optical transmittance, and shows excellent antireflective, antifogging, and self-cleaning properties, which is superior to the flat PDMS film (FPF). Therefore, we anticipate that the BSPF with extraordinary physicochemical properties may be an instruction for more scientists and engineers to focus on mimicking natural structures. It can also provide useful information to design more ingenious engineering products with desirable functionalities in the future.

\section{Experimental Section}

\subsection{Materials and Chemical Reagents}

Acetone, anhydrous ethanol, deionized water, methylene blue powders, and carbon black nanoparticles were purchased from Beijing Chemical Factory. The negative photoresist SU-8 2005 and its developer were obtained from the MicroChem Corp (Boston, MA, USA). The elastomer PDMS Sylgard 184 was purchased from Dow Corning. Trimethoxy $(1 \mathrm{H}, 1 \mathrm{H}, 2 \mathrm{H}, 2 \mathrm{H}$-heptadecafluorodecyl) silane was purchased from Aladdin (Saint Louis, MO, USA). Krytox 1506 lubricant was obtained from DuPont (Dover, DE, USA).

\subsection{Preparation of the Negative Structure Arrays (NSAs)}

The procedures were as follows: glass slides (approximately $4 \times 3 \mathrm{~cm}^{2}$ ) were processed separately with acetone, ethanol, and deionized water in an ultrasonic bath for $10 \mathrm{~min}$, after which the substrate was treated by plasma cleaner for $30 \mathrm{~s}$. SU-8 photoresist was coated on a glass substrate by a spin-coating program (500 rpm for $10 \mathrm{~s}$ with spinning acceleration of $200 \mathrm{rpm} / \mathrm{s}$, next, $3000 \mathrm{rpm}$ for $30 \mathrm{~s}$ with spinning acceleration of $500 \mathrm{rpm} / \mathrm{s}$ ), which was baked at $98{ }^{\circ} \mathrm{C}$ for $6 \mathrm{~min}$. Moreover, UV exposure (exposure energy at $158 \mathrm{~mJ} / \mathrm{cm}^{2}$ for $10 \mathrm{~s})$ was performed vertically to pattern of square-shaped arrays. Afterwards, a post bake was conducted at $95^{\circ} \mathrm{C}$ for $4 \mathrm{~min}$. Finally, the substrate was immersed in the developer solution for $100 \mathrm{~s}$.

\subsection{Preparation of the Papillae-Like Microstructure Arrays (PMAs)}

The processes were as follows: the pre-polymer and the curing agent were agitated soothingly (weight ratio 10:1). Second, the mixture was poured over the photoresist layer for the second-step replica of the positive microstructures. And the assembly was moved into the vacuum chamber for $40 \mathrm{~min}$ to remove air bubbles, next heated in a drying oven at $80^{\circ} \mathrm{C}$ for $1 \mathrm{~h}$ to cure completely the PDMS.

\subsection{Preparation of the Biomimetic Slippery PDMS Film (BSPF)}

The solidified PDMS was peeled off the substrate, and then the sample surface was cleaned using oxygen plasma. Subsequently, the sample was silanized using trimethoxy $(1 \mathrm{H}, 1 \mathrm{H}, 2 \mathrm{H}, 2 \mathrm{H}$-heptadecafluorodecyl) silane. Afterward, the Krytox 1506 lubricant was 
coated on the silanized biomimetic PDMS film (BPF) surface using a spin coater (SC100-SE) (Jiangsu Zeyuan Biotechnology Co. Ltd, Jiangyin, China).

\subsection{Characteristics of the Prepared Samples}

The surface morphology of the fabricated sample was observed by means of FESEM (JSM-6700LF, JEOL, Tokyo, Japan) at an accelerating voltage of $20 \mathrm{kV}$, a working distance of $14.1 \mathrm{~mm}$ and observation mode (low magnification, SE2). Laser scanning confocal microscope (LSCM) (LEXT OLS3000, Olympus, Tokyo, Japan) was used to observe the surface pattern of photoresist layer on the substrate. The static water contact angles of on the sample surfaces were estimated with an optical contact angle measuring device (OCA20 data physics, Filderstadt, Germany), a droplet $(3 \mu \mathrm{L})$ was dropped on four different surfaces in turn based on a sessile drop technique. The sliding angle was measured by inclining the sample until the droplet start sliding, and each sample was measured five times at room temperature. The chemical composition of the samples was characterized by using energy dispersive spectrometer (EDS, Oxford, UK) and X-ray photoelectron spectroscopy (XPS, SPECSXR50, Tokyo, Japan). The reflectance and transmittance spectra of the samples were obtained using a miniature fiber-optic spectrometer (Ocean Optics, Tallahassee, FL, USA) at normal incidence over a wavelength range of $400-800 \mathrm{~nm}$.

\subsection{Observation of Fog Drops Condensation}

The steady continuous fog was generated and controlled by an ultrasonic humidifier at a constant flow rate of $0.5 \mathrm{~mL} \cdot \mathrm{min}^{-1}$. After the surfaces were sprayed, at the same time, the macroscopic dynamic behavior of fog drops movement on the samples surface was observed by the single-lens reflex camera (Canon 80D, Tokyo, Japan). The laboratory temperature was $29^{\circ} \mathrm{C}$ and the relative humidity was $40 \%$.

\section{Results and Discussion}

In this work, we designed the surface topographies taking the form of a regular papillae-like microstructure arrays (PMAs) inspired from lotus leave on the PDMS substrate. The overall process of preparation was shown in Figure 1. Figure 2 shows 3D LSCM images of the negative structure arrays (NSAs) and the corresponding profile picture. Figure $3 \mathrm{a}$ is the microscale papillae surface of the lotus leaf in nature, based on the shape and sizes of its features, we firstly manufactured the negative structure arrays (NSAs) as shown in Figure 2. Here, we didn't choose the real lotus leaf as the template because its brittleness. Fortunately, we had confirmed that the UV lithography combined with a soft replication worked successfully to achieve the PDMS film with PMAs. Figure $3 \mathrm{~b}$ shows $45^{\circ}$-tilted oblique-view FESEM of the PMAs under 1000-fold magnification, which demonstrates overall morphology of the PMAs-based BPF. Figure $3 c$ shows enlarged FESEM image under 2000-fold magnification, further demonstrating the high quality and uniformity for biomimetic PMAs. A 3D image of the PMAs, as illustrated in Figure 3d. The width of the individual papillae $(\mathrm{W})$ is $6 \mu \mathrm{m}$, the pitch between neighboring papillaes $(\mathrm{P})$ is $10 \mu \mathrm{m}$, and the nominal height of the individual papillae $(\mathrm{H})$ is $6.5 \mu \mathrm{m}$.

Wettability is the affinity of liquid-solid surface, several theories have been introduced to describe the wettability of solid surface. In fact, to regulate the wettability and then the AF performance, surface roughness and composition need to be appropriately tailored.

Surface wettability on a perfectly flat, rigid, and chemically homogeneous solid surface is governed by Young's equation: 


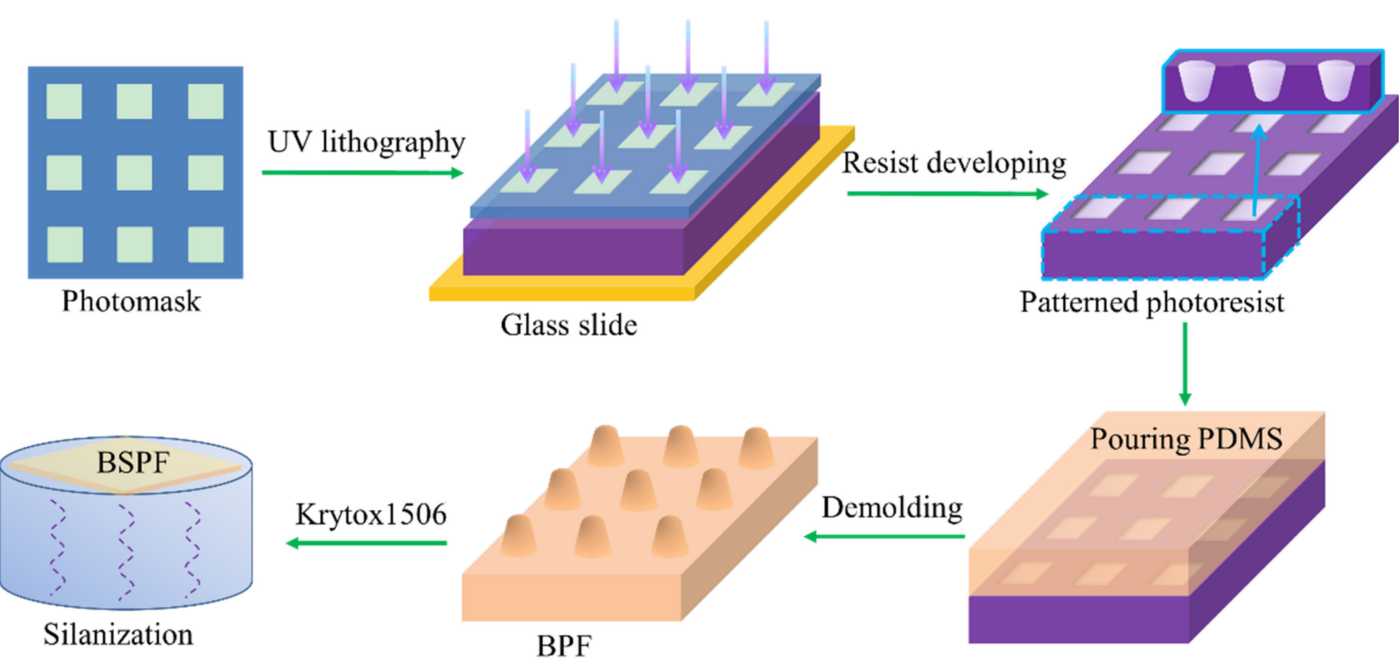

Figure 1. Schematic description for the fabrication of the BSPF surface.
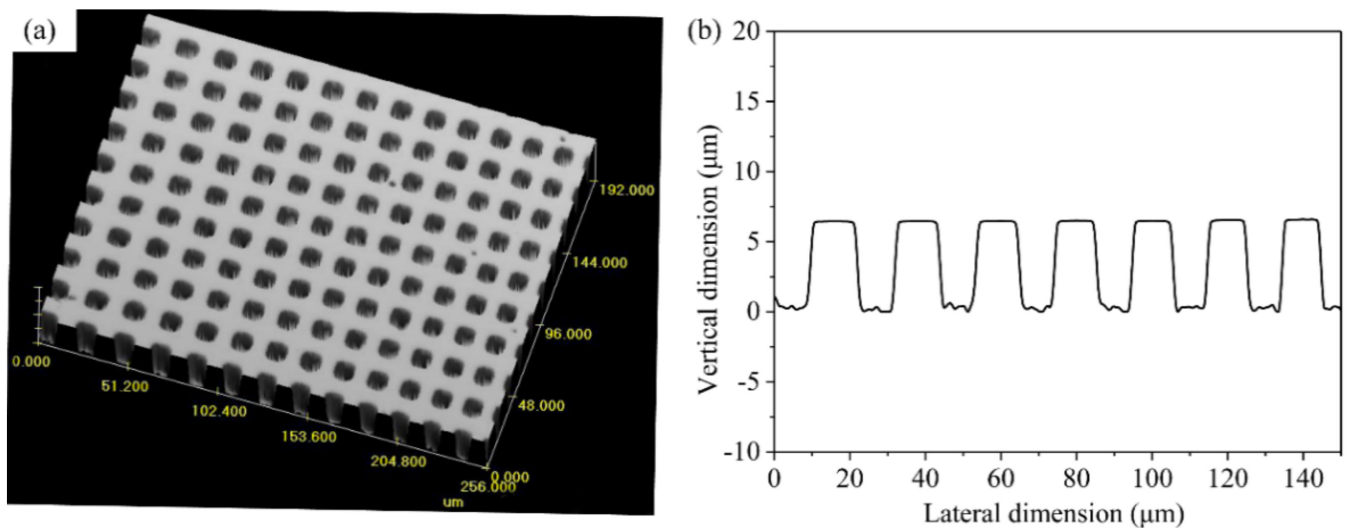

Figure 2. (a) 3D LSCM images of the negative structure arrays (NSAs); (b) the profile picture corresponding to (a).

$$
\gamma_{\mathrm{sv}}=\gamma_{\mathrm{sw}}+\gamma_{\mathrm{wv}} \cos \theta_{0}
$$

where $\gamma_{\mathrm{sv}}, \gamma_{\mathrm{sw}}$, and $\gamma_{\mathrm{wv}}$ are the interfacial tensions of solid-vapor, solid-water, and watervapor, respectively, $\theta_{0}$ is the static contact angle (CA) of the droplet on the ideally flat solid surface (Figure 4a). By infusing a liquid lubricant into a textured surface, the liquid overcoat forms a homogeneous slippery surface [30]. Thus, the modified Young's equation can be described as [24]:

$$
\gamma_{\mathrm{lv}}=\gamma_{\mathrm{lw}}+\gamma_{\mathrm{wv}} \cos \theta_{0}
$$

where $\gamma_{l_{v}}, \gamma_{l_{w}}$, and $\gamma_{\mathrm{wv}}$ are the interfacial tensions of lubricant-vapor, lubricant-water, and water-vapor, respectively, $\theta$ is the apparent CA of a water droplet on the liquid-infused surface (Figure $4 \mathrm{~b}$ ). In most cases, surface tension depends on surface chemistry, to a certain extent, while surface roughness allows for is modified, and therefore the wettability is determined strictly. Here, on the basis of the modified Young's equation, creating a hydrophobic surface (that is, $\theta>90^{\circ}$ ) would require to $\gamma_{l_{v}}$ be smaller than $\gamma_{l w}$ (that is, $\left.\gamma_{\mathrm{lv}}<\gamma_{\mathrm{lw}}\right)$.

Hence, we investigated the wetting properties of the flat PDMS film (FPF), biomimetic PDMS film (BPF), flat slippery PDMS film (FSPF) and biomimetic slippery PDMS film (BSPF) separately as shown in Figure 5a. For the FPF, a photograph of a water droplet was also shown in Figure $5 b$, exhibiting a hydrophobic behavior similarly to Figure 4c, i.e., a water contact angle $\left(\theta_{\mathrm{CA}}\right)$ of $\approx 105^{\circ}$. 


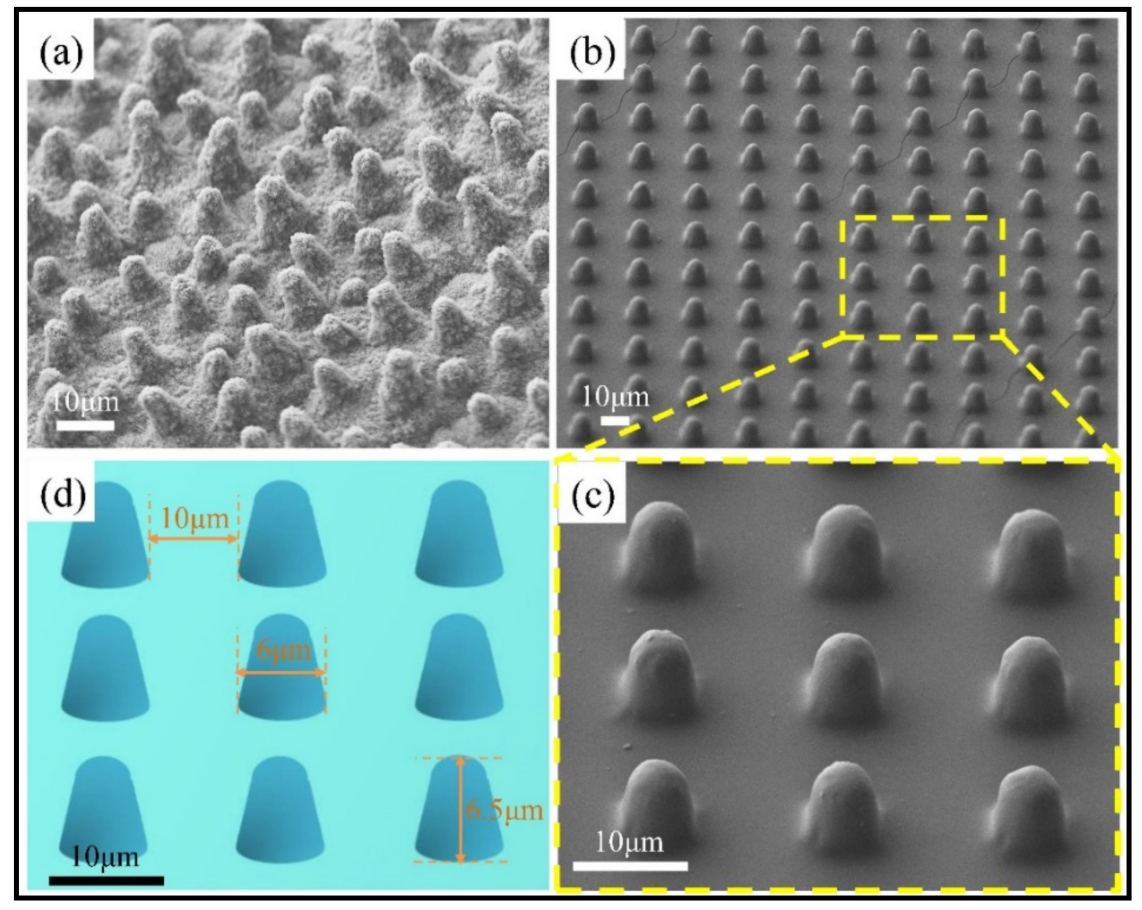

Figure 3. (a) The microscale papillae surface of the lotus leaf; (b) low-magnification FESEM image demonstrates overall morphology of the PMAs-based BPF; (c) enlarged FESEM image further demonstrates the high quality and uniformity for microstructures of the biomimetic PMAs; (d) a 3D image of the PMAs illustrates the global layout of the PMAs.

(a)

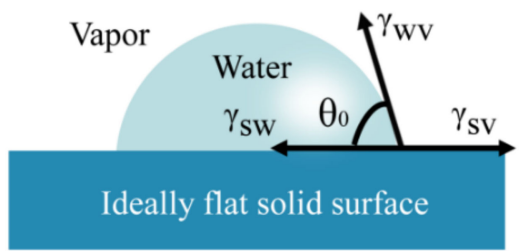

(c)

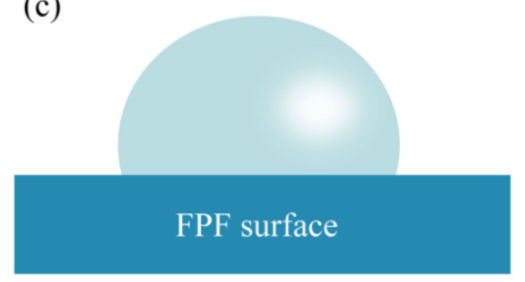

(b)

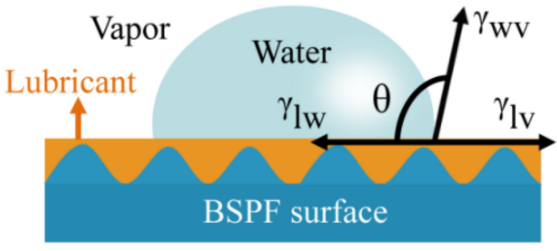

(d)

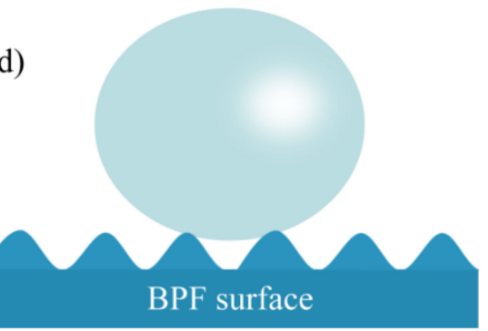

Figure 4. Schematic showing a liquid droplet sitting on an ideally flat solid surface (a), a BSPF surface (b), FPF surface (c) and BPF surface (d).

However, the surface presented high adhesive pinning state, where a water droplet is still tied to the surface even when the film is perpendicular. This is caused by the high adhesion of PDMS itself. Intuitively, Video S1 in the supporting information shows the sliding angle $\left(\theta_{S A}\right)$ of four sample surfaces. For the BPF, the hydrophobicity was enhanced remarkably similarly to Figure $4 \mathrm{~d}\left(\theta_{\mathrm{CA}} \approx 145^{\circ}\right.$ with a $\theta_{\mathrm{SA}}$ about $\left.55^{\circ}\right)$. This effect is attributed to the PMAs on the surface of PDMS film, which can be explained by the Cassie-Baxter model [31]. However, the $\theta_{\mathrm{CA}}$ value is lower than lotus leaves in 
nature (i.e., $\theta_{\mathrm{CA}} \geq 150^{\circ}$ and $\theta_{\mathrm{SA}} \leq 5-10^{\circ}$, superhydrophobicity). This is because there is no nanostructure on the surface [32].

(a)

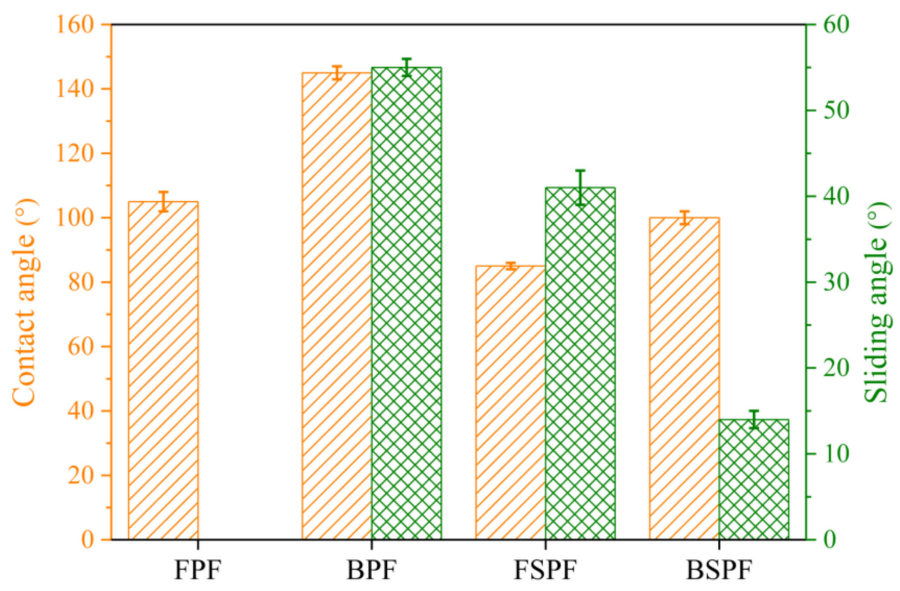

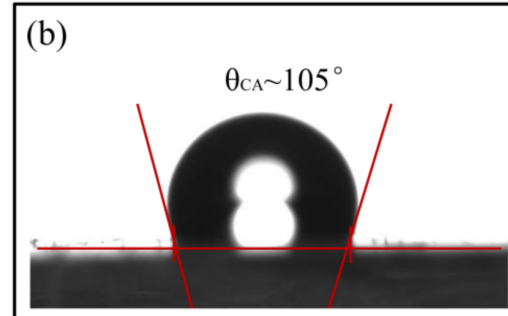

FPF

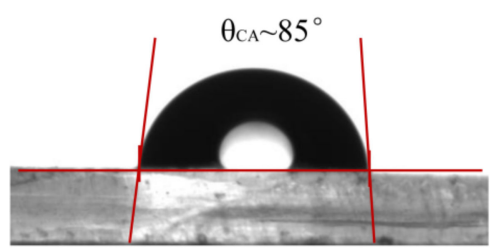

FSPF

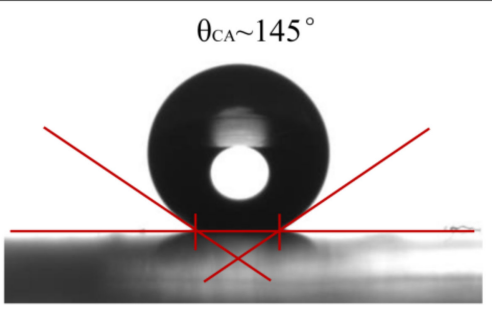

BPF

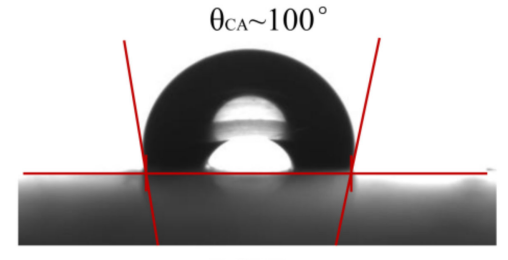

BSPF

Figure 5. (a) $\theta_{\mathrm{CA}}$ and $\theta_{\mathrm{SA}}$ measurements of four different surfaces; (b) optical images showing a macroscopic water droplet on the corresponding samples, $\theta_{\mathrm{CA}}$ is a water contact angle.

As a contrast, for the FSPF, though the hydrophobicity was decreased slightly with the $\theta_{\mathrm{CA}}$ value of about $85^{\circ}$, the surface adhesion was reduced obviously with $\theta_{\mathrm{SA}} \approx 41^{\circ}$. Especially, for the BSPF, the hydrophobicity was decreased dramatically relative to the $\mathrm{BPF}$, the $\theta_{\mathrm{CA}}$ value of $\approx 100^{\circ}$ even is lower than the FPF, but is higher than the FSPF. After the BPF surface was infused by Krytox 1506, the PMAs of the BPF surface was swelled and occupied by lubricant, giving rise to form a homogeneous smooth film on the BSPF surface. This smooth liquid film governed that the $\theta_{\mathrm{CA}}$ of BSPF surface can barely more than $120^{\circ}$, since the limitation of interfacial tension between lubricant and water [33]. Additionally, broadly speaking, a hydrophilic surface displays a strong affinity for water, while a hydrophobic surface tends to repel it. As can be easily understood, the BSPF surface present a low adhesion state with a $\theta_{\text {SA }}$ less than $15^{\circ}$.

To better clarify why BSPF surface possessed above wettability behavior. We firstly investigated the differences in the chemical compositions of the BPF and the BSPF by energy dispersive spectrometer (EDS). EDS results indicated that the percentage of $\mathrm{C}, \mathrm{O}$, and $\mathrm{Si}$ atoms for BPF sample was 37.33, 35.68, and 26.99, respectively. Furthermore, it was seen that upon Krytox 1506 treated BPF surface (i.e., BSPF), the total content of F atom on the surface was 26.14, and the $\mathrm{O}$ atom correspondingly decrease to $17.79 \%$ (Figure 6). In addition, we also performed an X-ray photoelectron spectroscopy (XPS) analysis at 
each step toward the BPF and the as-fabricated BSPF, respectively (Figure 7). The BPF surface appear the $\mathrm{C} 1 \mathrm{~s}(283.8 \mathrm{eV}), \mathrm{O} 1 \mathrm{~s}(531.7 \mathrm{eV})$, and $\mathrm{Si} 2 \mathrm{p}(102.6 \mathrm{eV})$ peaks, as shown in Figure $7 \mathrm{a}$.
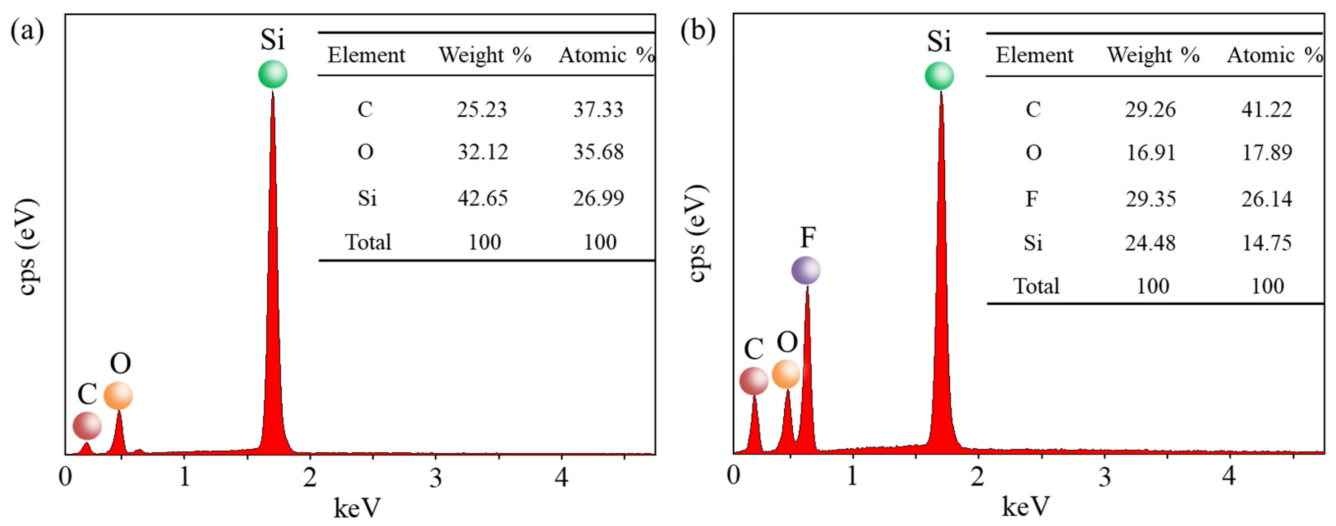

Figure 6. Chemical compositions of the BPF and BSPF. (a) EDS spectrum indicates that the main elements of BPF are $\mathrm{C}, \mathrm{O}$ and $\mathrm{Si}$; (b) while the main elements of BSPF are C, O, F and Si.

(a)
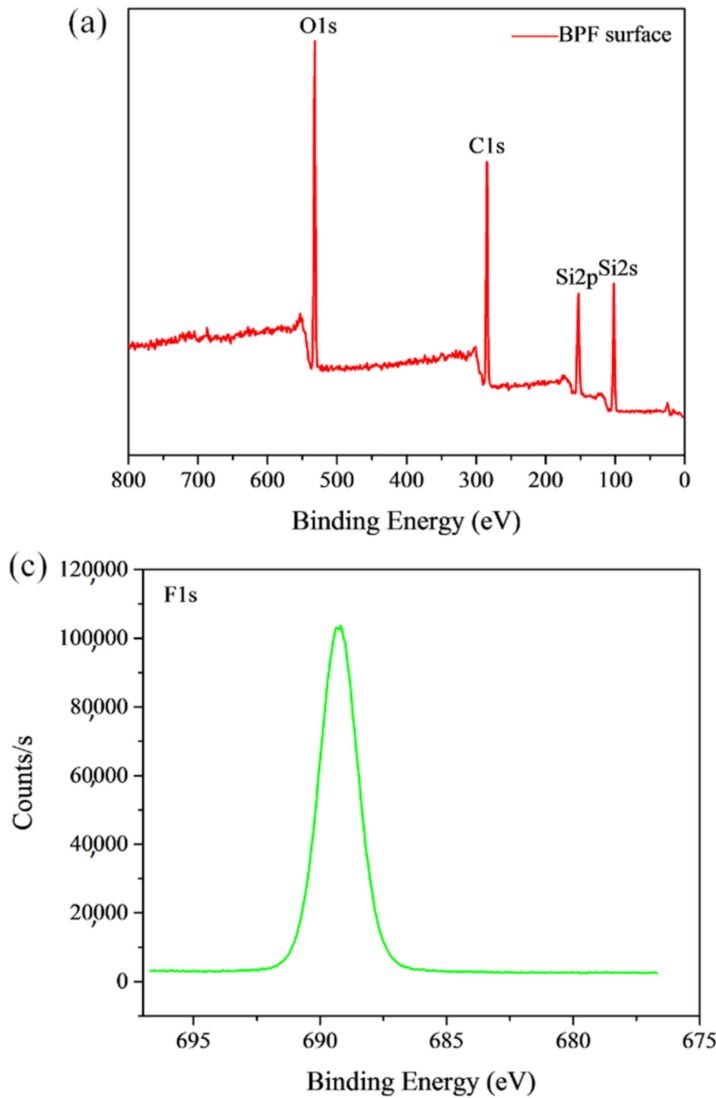

(b)

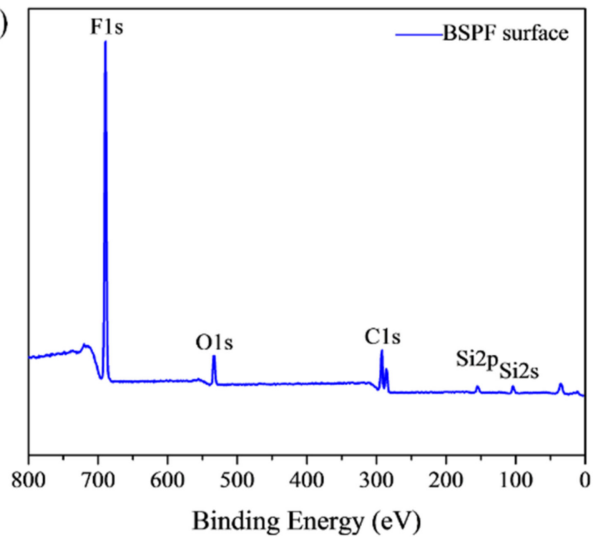

(d)

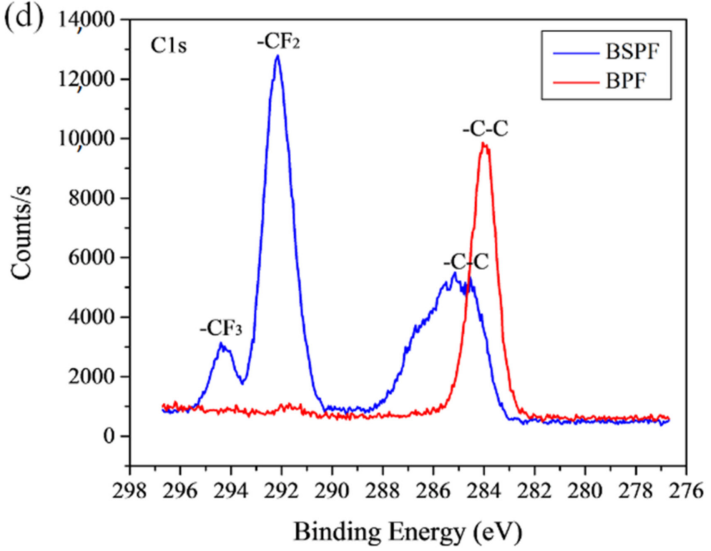

Figure 7. XPS spectrum results of (a) the analysis of BPF surface; (b) the analysis of BSPF surface; (c) the high-resolution F 1s peak of BSPF surface; (d) the high-resolution C 1s peak of BPF (red) and BSPF (blue) surface.

However, the $\mathrm{C} 1 \mathrm{~s}$ peak occurred at the binding energy of $291.8 \mathrm{eV}$ on the BSPF surface (Figure $7 \mathrm{~b})$. The fluorinated alkyl can be found by an apparently F $1 \mathrm{~s}(688.6 \mathrm{eV})$ peak (Figure 7c). Figure 7d showed the high-resolution $C$ 1s peak was decomposed into three component peaks, including $-\mathrm{C}-\mathrm{C} / \mathrm{C}-\mathrm{H}(283.8 \mathrm{eV}),-\mathrm{CF}_{2}(290.6 \mathrm{eV})$, and $-\mathrm{CF}_{3}$ $(293.7 \mathrm{eV})$. For the lowest surface energy groups, which have an order of $-\mathrm{CH}_{2}->-\mathrm{CH}_{3}$ 
$>-\mathrm{CF}_{2}->-\mathrm{CF}_{2} \mathrm{H}>-\mathrm{CF}_{3}$ [34]. Thus, the BSPF surface has lower the surface energy due to its high content of $-\mathrm{CF}_{3},-\mathrm{CF}_{2}-$ groups. Moreover, the film exhibits lower surface adhesion due to the lower surface energy. Consequently, the existence of $-\mathrm{CF}_{2}-$ group is crucial, lead to lowering the surface adhesion. For this reason, the BSPF surface have dramatically lower $\theta_{\mathrm{SA}}$ than three other surfaces.

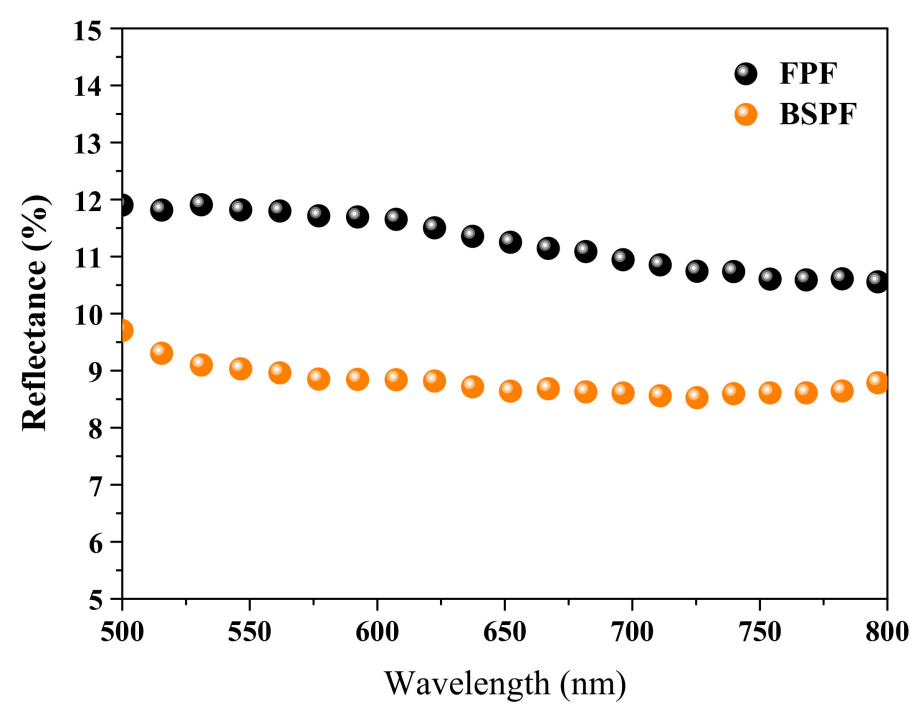

Figure 8. Reflectance spectra of the FPF (black) and the BSPF (orange).

To analyze the optical characteristics of the BSPF, the original reflectance and original transmittance spectra of the FPF (reference substance) and BSPF were respectively measured. As shown in Figure 8, it can be seen that the BSPF displayed a broadband reduction in reflectance for the major visible wavelengths (400 to $800 \mathrm{~nm}$ ), in comparison to the FPF. An average reflection of $\sim 11.3 \%$ of the FPF was reduced to $\sim 8.9 \%$ in the BSPF due to the presence of PMAs, the results show that reflection of roughed front surface was decreased result from augmentation of the effective index of the PMAs. However, the smooth bottom interface of the BSPF surface also may cause reflection, and the air was not remarkably influenced by the front surface structure. As a result, the total reflection of the BSPF surface would not extraordinarily decline than that FPF surface. As shown in Figure 9a, each transmittance spectrum was obtained over all wavelengths of interest and the BSPF shows a maximal transmittance of $90.5 \%$ in the long wave band $(700-800 \mathrm{~nm})$. The total transmittance spectrum of the BSPF is obviously lower than that of the FPF $(87.6 \%)$ over wavelengths range of $400-580 \mathrm{~nm}$, while it is basically consistent at $580-700 \mathrm{~nm}$ wavelengths range. In the wavelength region of $400-580 \mathrm{~nm}$, the slippery bottom interface of the BSPF may increase mirror reflection losses for incident light at the corresponding wavelengths. In addition, the PMAs exhibit a nearly linearly graded effective refractive index distribution from air to the PDMS, which can efficiently reduce the surface reflection. Thus, this transmission of the BSPF can further increase in the wavelength range of 580-700 nm, until a wavelength range of 700-800 nm, both the FPF and BSPF samples show the very similar average transmission of $\sim 85.6$ and $87.5 \%$, respectively. In fact, the light capture effects of the PMAs is indeed the key point to realize the antireflective strategy, to clarify the relationship between geometrical parameters and optical property, different geometrical sizes (width, gap, height or aspect ratio) of PMAs structure need to be designed and fabricated to further optical analysis in future work.

To investigate the antifogging property of the BSPF, the generated fog was consecutively sprayed on the BSPF surface, and the spraying timer gradually increased within $90 \mathrm{~s}$ at an interval of $30 \mathrm{~s}$, the transmittance spectra changes was observed within the wavelength range from 400 to $800 \mathrm{~nm}$. The BSPF was blow-dried after the transmittance spectrum was obtained each time, which eventually formed a dynamic cyclical process of 
"spray-blow-dry-spray". After the BSPF was consecutively sprayed by the generated fog for the $30 \mathrm{~s}$ and $60 \mathrm{~s}$, as shown in Figure $9 \mathrm{~b}, \mathrm{c}$, the maximal transmittance in the short wave band (400-500 nm) always remain below $60 \%$, within the wavelength range from 500 to $800 \mathrm{~nm}$, the maximal transmittances reached around $80 \%$. However, with the increase of fog amount, the BSPF began to occur scattering losses for incident light at the corresponding wavelengths. Hence, the transmittance decreased to below $78 \%$. Interestingly, after the BSPF was consecutively sprayed by the generated fog for about $90 \mathrm{~s}$, as shown in Figure $9 \mathrm{~d}$, the maximal transmittance reached over $60 \%$ in the short wave band (400-500 nm), and the maximal transmittance was close to $90 \%$ in the long wave band (500-800 nm). The transmittance spectra have a similar variation trend within the $90 \mathrm{~s}$ gradient spray over the major visible wavelengths $(400-800 \mathrm{~nm})$. The transmittance basically recovered to the original state, which is particularly distinct in the long wavelength (500-800 nm). It can be identified that the PMAs-based BSPF has outstanding AF ability.
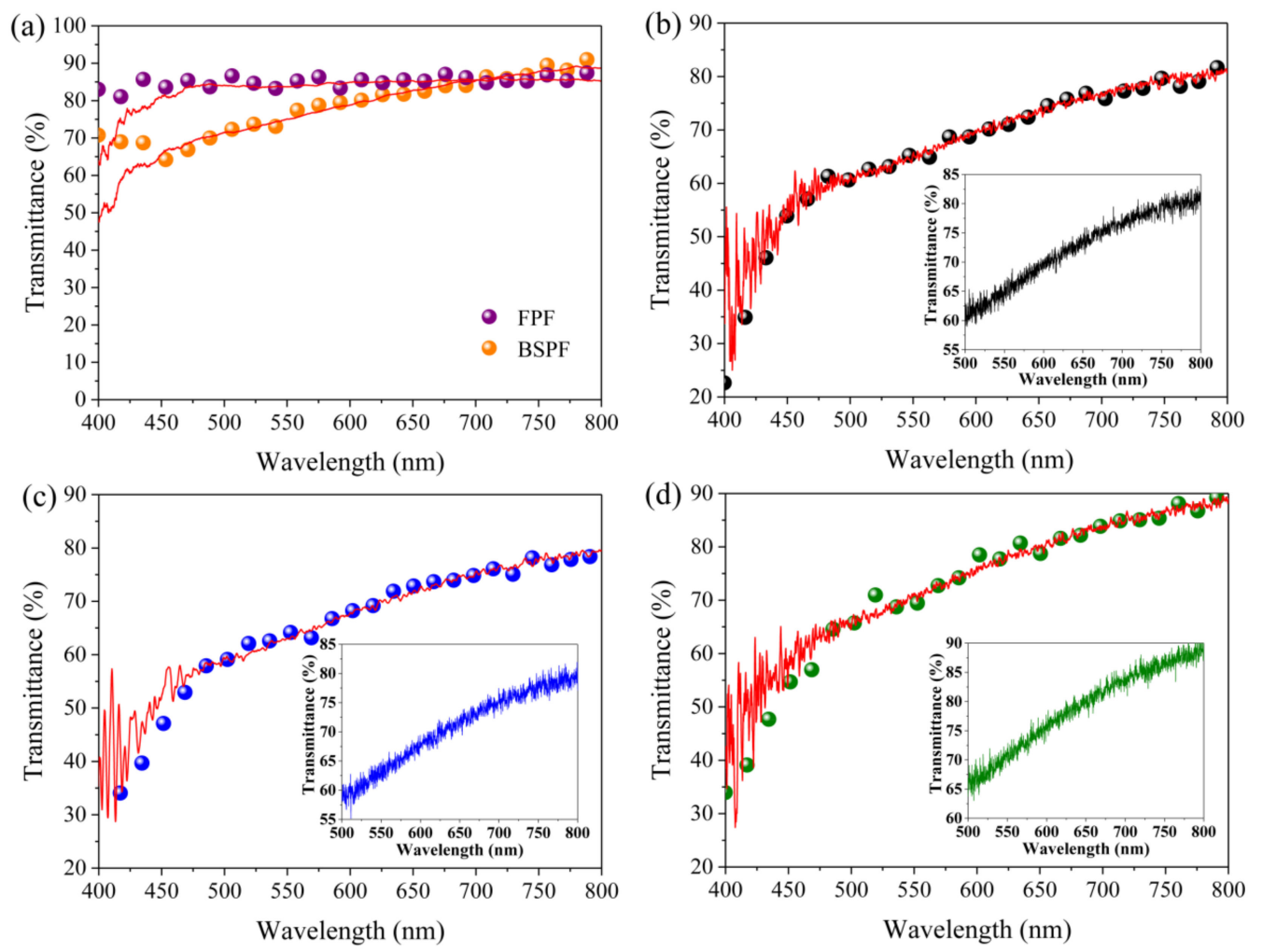

Figure 9. (a) Initial transmittance spectra of the FPF (purple) and BSPF (orange) before spray simulation; (b) transmittance of the BSPF changes after spray simulation for $30 \mathrm{~s}$; (c) transmittance of the BSPF changes after spray simulation for $60 \mathrm{~s}$; (d) transmittance recovers to initial fog-free state after continuous spray simulation for $90 \mathrm{~s}$.

Additionally, to verify the AF behavior of BSPF surface more intuitively, the macrodynamic process of fog drops movement was recorded using a single-lens reflex camera (Video S2). As shown in Figure 10a, as time gone on, we surprisingly discovered that some tiny fog drops started to merge with each other and form larger fog droplet (see the same color outline from $t=20 \mathrm{~s}$ to $t=90 \mathrm{~s}$ ). Furthermore, when the mist droplets reached a certain size, larger fog drops began to slide off the BSPF by gravity-driven. Ultimately, this slide-off behavior taken away some tiny fog drops along the way, sweeping the surface clean and retaining the area dry (see orange heart-shaped outline at $t=120 \mathrm{~s}$ ). This phenomenon was exactly consistent with the results of transmittance spectra, further demonstrating the excellent antifogging property of the BSPF surface. However, during this spray fog process, it is not hard to find that the tiny mist droplet did not easily slip on the BSPF surface, and cannot be overwhelmed by its gravity. It suggests that the antifogging behavior of the BSPF 
surface seems imperfect. Figure 10b shows the antifogging efficiency (i.e., the ratios of fog removal area to the total area) on the resultant surfaces. On the whole, the BSPF surface continuously presents superior antifogging effectiveness compared with the other three surfaces. After $120 \mathrm{~s}$, the maximum antifogging efficiency is close to $100 \%$ for the BSPF surface, while the antifogging efficiency for FPF, BPF and FSPF are about 35\%, 70\% and $85 \%$, respectively.
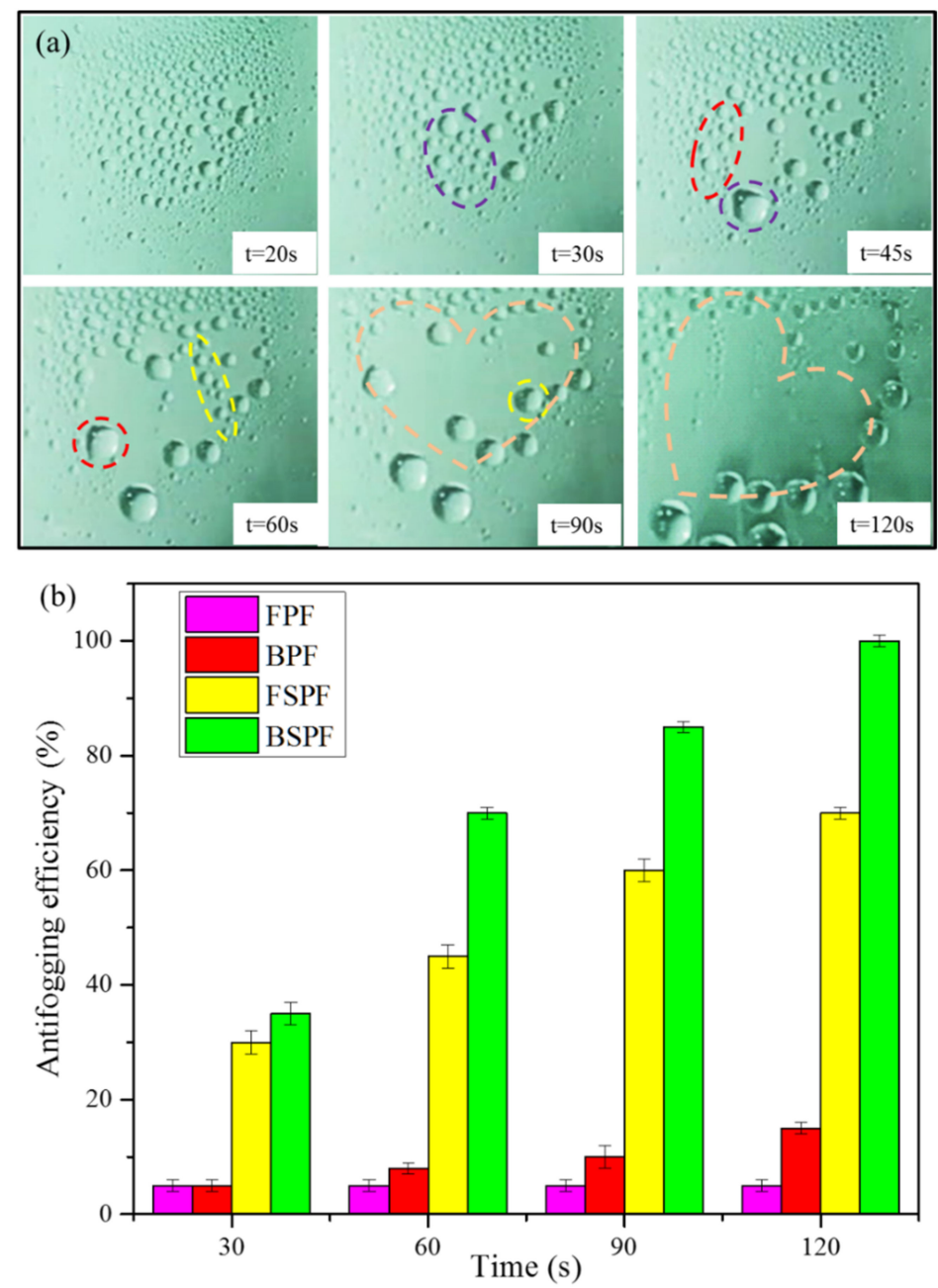

Figure 10. (a) Optical images show that the macro-dynamic process of fog drops movement on the BSPF surface. From $t=20 \mathrm{~s}$ to $t=120 \mathrm{~s}$, with water condensation, some tiny fog drops began to merge with each other and form larger fog drops (the same color outline from $t=20 \mathrm{~s}$ to $t=120 \mathrm{~s}$ ). As the fog drops reach a certain size, the fog drops begin to slide off the BSPF by gravity-driven and take away some tiny fog drops along the way (see orange heart-shaped outline at $t=120 \mathrm{~s}) ;(\mathbf{b})$ the graph of antifogging efficiency on the resultant surfaces. At $t=120 \mathrm{~s}$, the maximum antifogging efficiency for BSPF surface is close to $100 \%$, while the antifogging efficiency for FPF, BPF and FSPF are about $35 \%, 70 \%$ and $85 \%$, respectively.

The self-cleaning ability of transparent materials that integrate high transparency (antireflection) and antifogging properties are widely useful and very imperative in daily life. In order to estimate the self-cleaning performance of the BSPF, samples of both the FPF and BSPF were stuck on glass slide, and contaminated with the hydrophilic methylene blue (MB) powders, and hydrophobic carbon black (CB) nanoparticles, separately. The amounts of contaminants on the samples surface were used to assess the self-cleaning ability before and after rinsing. First, the both surfaces were deliberately contaminated by dust, then some water droplets were dropped onto the both surfaces. After droplet rinsing, 
the optical microscopy images of hydrophilic contaminants on the FPF and BSPF were shown in Figure 11b. It can be noticed that on the BSPF surface where MB powders can first be dissolved in water and then slide off the surfaces, for better a view of the clean and transparent surface, please see the supplement Video S3-S5. In particular, the bottom of the petri dish is full of blue obviously, further demonstrating that the MB powders on BSPF surface are efficiently cleared off. Whereas for the FPF surface, the MB powders tended to accumulate at the air/water interface. As a result, hydrophilic powders adhere to the FPF surface. As a contrast, Figure 11c shows hydrophobic contaminants on the FPF and BSPF after rinsing with droplets, respectively. Significantly, although the BSPF surface has much less contamination than the FPF surface under water flow, there was still a small amount of hydrophobic dust attached to the BSPF surface. Competitive absorption of Krytox 1506 generated between $\mathrm{CB}$ and the BSPF surface, leading to the high surface affinity of CB. Likewise, hydrophilic viscous ketchup was also regarded as a liquid contaminant, which can also be removed from the BSPF surface with ease using water drops (Figure 11d), further confirming the excellent self-cleaning ability of the BSPF surface. Based on the contrast for the self-cleaning performance, we discover that the BSPF surface exhibited a better self-cleaning ability toward a variety of liquids than solid dust. However, the residual of solid contaminants on BSPF for the rinsing conditions is might due to the formation of "water-spotting" effects that localize the particles on the surface. The self-cleaning ability of the BSPF in mild rain condition is an encouraging prospect for outdoor applications, especially for heavy rain condition, since the rinsing may be like mild rain events.
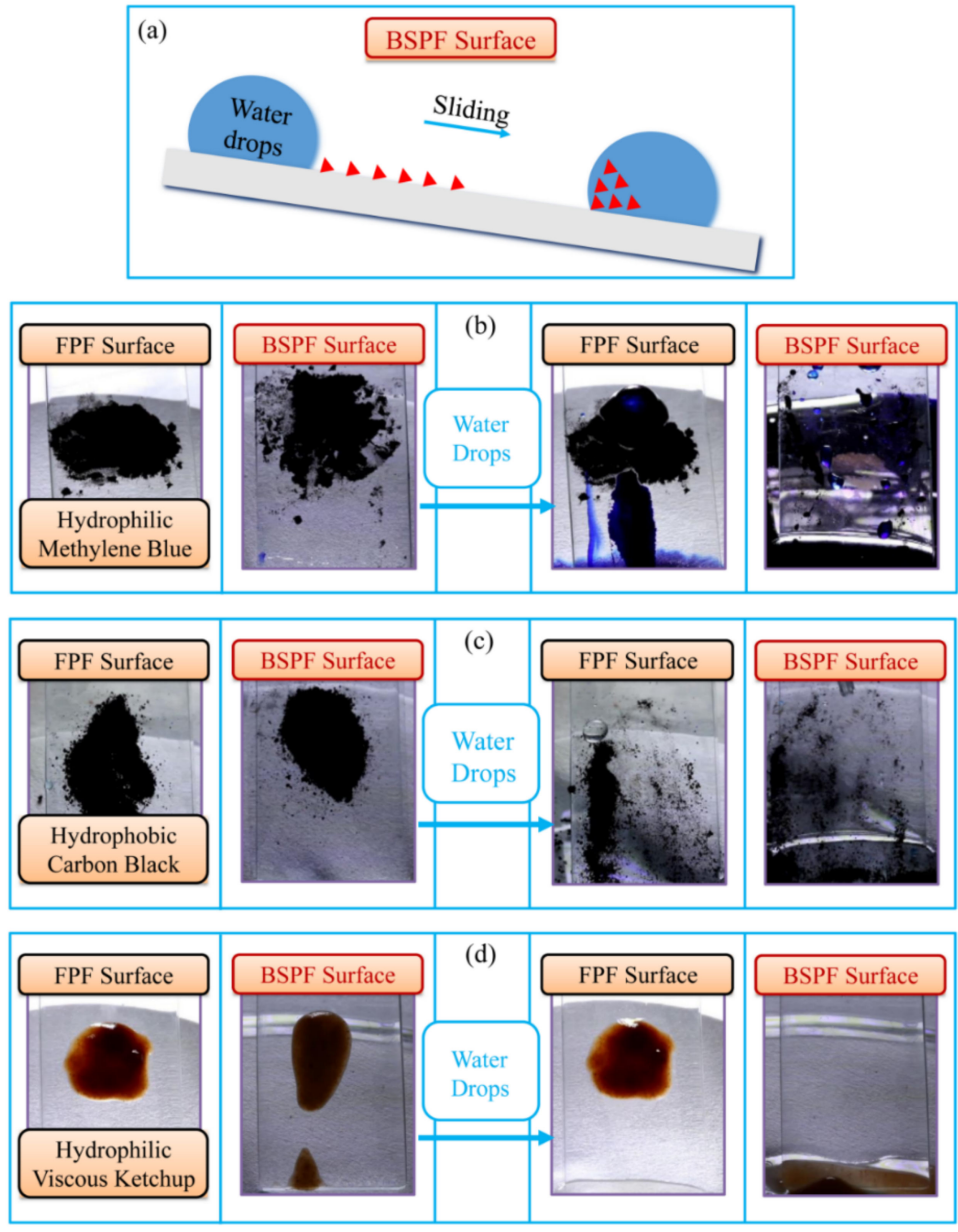

Figure 11. (a) Cleaning mechanism of BSPF surface defined as the "slide-to-clean" strategy; the self-cleaning ability of the three surfaces was tested by (b) hydrophilic dust (MB powders), (c) hydrophobic dust (CB nanoparticles) and (d) hydrophilic liquid (viscous ketchup). 
In addition, we also found different tilt angles will produce different self-cleaning effects, the maximal tilt angle is $30^{\circ}$ for the self-cleaning ability of BSPF in this paper, and the long-term stability of the Krytox under constant continuous long-term exposure to sunlight has not been tested, it's still a big challenge to be applied to solar cells. Therefore, in the following work, we will verify the mechanical stability of the BSPF follow up the Sun's trajectory across the sky, and hope it can be applied in practical application soon.

\section{Conclusions}

In this work, a BSPF was designed and fabricated successfully via a common UV lithography combined with a soft replication, subsequently infused with Krytox 1506 lubricant. The resultant surface has hydrophobic surface chemistry, a slippery interface, PMAs structure. Then, the wettability, optical characteristic, antifogging property and self-cleaning ability of the PMAs-based BSPF were characterized experimentally. The BSPF exhibits excellent antifogging and self-cleaning properties, which is superior to the FPF. Remarkably, an average reflection of $\sim 11.3 \%$ in the FPF was reduced to $\sim 8.9 \%$ of the BSPF. In addition, after gradient spray test for $120 \mathrm{~s}$, the antifogging efficiency was close to $100 \%$ for the BSPF surface compared with the other surfaces (35\%, $70 \%$ and $85 \%)$. Further, we also discovered that the BSPF surface exhibited a better self-cleaning performance toward various liquids than solid dust. Notwithstanding some essential information on the BSPF surface such as the durability and mechanical strength has not been considered, the surface may offer more options for particular antifogging applications, such as solar cell installations, solar panels, and so forth. Therefore, we anticipate that the BSPF surface with extraordinary physicochemical properties may be an instruction for more scientists and engineers to focus on mimicking natural structures. It can also furnish favorable information on the design, preparation, and applications of antifogging materials on polymers and other materials substrate.

Supplementary Materials: The following are available online at https:/ /www.mdpi.com/2079-6 412/11/2/238/s1, Video S1: the sliding angle $\left(\theta_{\mathrm{SA}}\right)$ of four sample surfaces, Video S2: the macrodynamic process of fog drops movement, Video S3: the self-cleaning performance of the BSPF with the hydrophilic methylene blue (MB) powders, Video S4: the self-cleaning performance of the BSPF with the hydrophobic carbon black (CB) nanoparticles, Video S5: the self-cleaning performance of the BSPF with the hydrophilic viscous ketchup.

Author Contributions: Conceptualization, S.N. and Z.H.; data curation, S.N.; formal analysis, X.F.; funding acquisition, X.F., H.G., S.N. and Z.H.; investigation, H.G., Z.W.; methodology, S.N.; project administration, Z.H.; supervision, Z.H.; validation, X.F., H.G. and Z.W.; Visualization, H.G., Z.W.; writing—original draft preparation, X.F.; writing—review and editing, S.N., Z.H. All authors have read and agreed to the published version of the manuscript.

Funding: This work was supported by the National Natural Science Foundation of China (No. 52005227, 51805207, 51875244, 51835006), the Natural Science Research Project of Jiangsu Province University (No.20KJB410003), and the National Key Research and Development Program of China (No. 2018YFA0703300).

Data Availability Statement: The study did not report any data.

Conflicts of Interest: The authors declare no conflict of interest.

\section{References}

1. Li, Y.; Fang, X.; Wang, Y.; Ma, B.; Sun, J. Highly transparent and water-enabled healable antifogging and frost-resisting films based on poly (vinyl alcohol)-nafion complexes. Chem. Mater. 2016, 28, 6975-6984. [CrossRef]

2. Babeva, T. Special issue: Optical thin films and structures: Design and advanced applications. Coatings 2020, 10, 1140. [CrossRef]

3. Duran, I.R.; Laroche, G. Water drop-surface interactions as the basis for the design of anti-fogging surfaces: Theory, practice, and applications trends. Adv. Colloid Interface Sci. 2019, 263, 68-94. [CrossRef] [PubMed]

4. Chang, C.; Lin, Z.; Song, L.; Cheng, L. Preparation of organic-inorganic hybridized dual-functional antifog/antireflection coatings on plastic substrates. J. Appl. Polym. Sci. 2020, 137, 48822. [CrossRef] 
5. Domke, M.; Sonderegger, G.; Kostal, E.; Matylitsky, V.; Stroj, S. Transparent laser-structured glasses with superhydrophilic properties for anti-fogging applications. Appl. Phys. A 2019, 125, 675. [CrossRef]

6. Park, S.; Park, S.; Jang, D.H.; Lee, H.S.; Park, C.H. Anti-fogging behavior of water-absorbing polymer films derived from isosorbide-based epoxy resin. Mater. Lett. 2016, 180, 81-84. [CrossRef]

7. Yoon, J.; Ryu, M.; Kim, H.; Ahn, G.; Yim, S.; Kim, D.; Lee, H. Wet-style superhydrophobic antifogging coatings for optical sensors. Adv. Mater. 2020, 32, 2002710. [CrossRef] [PubMed]

8. Tsui, K.H.; Lin, Q.; Chou, H.; Zhang, Q.; Fu, H.; Qi, P.; Fan, Z. Low-cost, flexible, and self-cleaning 3D nanocone anti-reflection films for high-efficiency photovoltaics. Adv. Mater. 2014, 26, 2805-2811. [CrossRef] [PubMed]

9. Ghymn, Y.H.; Jung, K.; Shin, M.; Ko, H. A luminescent down-shifting and moth-eyed anti-reflective film for highly efficient photovoltaic devices. Nanoscale 2015, 7, 18642-18650. [CrossRef] [PubMed]

10. Ren, J.; Wang, Y.; Yao, Y.; Wang, Y.; Fei, X.; Qi, P.; Lin, S.; Kaplan, D.L.; Buehler, M.J.; Ling, S. Biological material interfaces as inspiration for mechanical and optical material designs. Chem. Rev. 2019, 119, 12279-12336. [CrossRef]

11. Han, Z.; Feng, X.; Guo, Z.; Niu, S.; Ren, L. Flourishing bioinspired antifogging materials with superwettability: Progresses and challenges. Adv. Mater. 2018, 30, 1704652. [CrossRef]

12. Wen, L.; Tian, Y.; Jiang, L. Bioinspired super-wettability from fundamental research to practical applications. Angezw Chem. Int. Edit. 2015, 54, 3387-3399. [CrossRef] [PubMed]

13. Yuan, J.; Yan, S.; Zhang, X. Superhydrophilic antifogging broadband antireflective coatings with worm-like nanostructures fabricated by one dip-coating method and calcination. Appl. Surf. Sci. 2020, 506, 144795. [CrossRef]

14. Gao, X.; Yan, X.; Yao, X.; Xu, L.; Zhang, K.; Zhang, J.; Yang, B.; Jiang, L. The dry-style antifogging properties of mosquito compound eyes and artificial analogues prepared by soft lithography. Adv. Mater. 2007, 19, 2213-2217. [CrossRef]

15. Sun, Z.; Liao, T.; Liu, K.; Jiang, L.; Kim, J.H.; Dou, S. Fly-eye inspired superhydrophobic anti-fogging inorganic nanostructures. Small 2014, 10, 3001-3006. [CrossRef]

16. Li, J.; Zhu, J.; Gao, X. Bio-inspired high-performance antireflection and antifogging polymer films. Small 2014, 10, $2578-2582$. [CrossRef] [PubMed]

17. Liu, C.; Ju, J.; Zheng, Y.; Jiang, L. Asymmetric ratchet effect for directional transport of fog drops on static and dynamic butterfly wings. ACS Nano 2014, 8, 1321-1329. [CrossRef]

18. Han, Z.; Mu, Z.; Li, B.; Wang, Z.; Zhang, J.; Niu, S.; Ren, L. Active antifogging property of monolayer $\mathrm{SiO}_{2}$ film with bioinspired multiscale hierarchical pagoda structures. ACS Nano 2016, 10, 8591-8602. [CrossRef] [PubMed]

19. Wang, Q.; Yao, X.; Liu, H.; Quéré, D.; Jiang, L. Self-removal of condensed water on the legs of water striders. Proc. Natl. Acad. Sci. USA 2015, 112, 9247-9252. [CrossRef]

20. Tsai, Y.C.; Shieh, J.J.A.S.S. Growing invisible silica nanowires on fused silica plates provides highly transparent and superwetting substrates. J. Ind. Eng. Chem. 2019, 479, 619-625. [CrossRef]

21. Wang, L.; Gong, Q.; Zhan, S.; Jiang, L.; Zheng, Y. Robust anti-icing performance of a flexible superhydrophobic surface. Adv. Mater. 2016, 28, 7729-7735. [CrossRef] [PubMed]

22. Yao, X.; Song, Y.; Jiang, L. Applications of bio-inspired special wettable surfaces. Adv. Mater. 2011, 23, 719-734. [CrossRef]

23. Cheng, Y.T.; Rodak, D.E. Is the lotus leaf superhydrophobic? J. Mater. Chem. 2005, 86, 144101. [CrossRef]

24. Dai, X.; Sun, N.; Nielsen, S.O.; Stogin, B.B.; Wang, J.; Yang, S.; Wong, T.S. Hydrophilic directional slippery rough surfaces for water harvesting. Sci. Adv. 2018, 4, eaaq0919. [CrossRef] [PubMed]

25. Miljkovic, N.; Enright, R.; Nam, Y.; Lopez, K.; Dou, N.; Sack, J.; Wang, E.N. Jumping-droplet-enhanced condensation on scalable superhydrophobic nanostructured surfaces. Nano Lett. 2012, 13, 179-187. [CrossRef] [PubMed]

26. Thompson, C.; Fleming, R.; Zou, M. Transparent self-cleaning and antifogging silica nanoparticle films. Sol. Energy Mat. Sol. C 2013, 115, 108-113. [CrossRef]

27. Raut, H.K.; Ranganath, A.S.; Baji, A.; Wood, K.L.J.A.S.S. Bio-inspired hierarchical topography for texture driven fog harvesting. Appl. Surf. Sci. 2019, 465, 362-368. [CrossRef]

28. Anand, S.; Paxson, A.T.; Dhiman, R.; Smith, J.D.; Varanasi, K.K. Enhanced condensation on lubricant-impregnated nanotextured surfaces. ACS Nano 2012, 6, 10122-10129. [CrossRef]

29. Luo, Y.; Li, J.; Zhu, J.; Zhao, Y.; Gao, X. Fabrication of condensate microdrop self-propelling porous films of cerium oxide nanoparticles on copper surfaces. Angew Chem. Int. Edit. 2015, 54, 4876-4879. [CrossRef] [PubMed]

30. Wong, T.S.; Kang, S.H.; Tang, S.K.; Smythe, E.J.; Hatton, B.D.; Grinthal, A.; Aizenberg, J. Bioinspired self-repairing slippery surfaces with pressure-stable omniphobicity. Nature 2011, 477, 443. [CrossRef]

31. Lv, C.; Zhang, X.; Niu, F.; He, F.; Hao, P. From initial nucleation to Cassie-Baxter state of condensed droplets on nanotextured superhydrophobic surfaces. Sci. Rep. 2017, 7, 42752. [CrossRef]

32. Bai, H.; Zhang, L.; Gu, D. Micrometer-sized spherulites as building blocks for lotus leaf-like superhydrophobic coatings. Appl. Surf. Sci. 2018, 459, 54-62. [CrossRef]

33. Cao, M.; Guo, D.; Yu, C.; Li, K.; Liu, M.; Jiang, L. Water-repellent properties of superhydrophobic and lubricant-infused "slippery" surfaces: A brief study on the functions and applications. ACS Appl. Mater. Interfaces 2015, 8, 3615-3623. [CrossRef] [PubMed]

34. Liu, Y.; Liu, J.; Li, S.; Liu, J.; Han, Z.; Ren, L. Biomimetic superhydrophobic surface of high adhesion fabricated with micronano binary structure on aluminum alloy. ACS Appl. Mater. Interfaces 2013, 5, 8907-8914. [CrossRef] [PubMed] 